\title{
Giant Viruses and their mobile genetic elements: the molecular symbiotic hypothesis.
}

\author{
Jonathan Filée
}

Evolution, Génomes, Comportement \& Ecologie, CNRS, IRD, Univ.Paris-Sud, Université Paris-Saclay, 91198 Gif-sur-Yvette

Avenue de la Terrasse, 91190 Gif sur Yvette, France.

jonathan.filee@egce.cnrs-gif.fr

\section{Highlight}

- Giant Virus (GV) genomes are loaded with diverse classes of mobile genetic elements (MGEs)

- MGEs cooperate with GV genes in order to fulfill viral functions.

- Site-specific endonucleases encoded by MGEs are used as anti-host or anti-competing viral compounds

- Integrase/transposase genes derived from MGEs have been recruited to generate integrative proviral forms.

- MGEs and GVs may thus compose a mutualistic symbiosis

\section{Summary}

Among the virus world, Giant viruses (GVs) compose one of the most successful eukaryovirus families. In contrast with other eukaryoviruses, GV genomes encode a wide array of mobile genetic elements (MGEs) that encompass diverse, mostly prokaryotic-like, transposable element families, introns, inteins, restriction-modification systems and enigmatic classes of mobile elements having little similarities with known families. Interestingly, several of these MGEs may be beneficial to the GVs, fulfilling two kinds of functions: 1) degrading host or competing virus/ virophages DNA and 2) promoting viral genome integration, dissemination and excision into the host genomes.

By providing fitness advantages to the virus in which they reside, these MGES compose a kind of molecular symbiotic association in which both partners should be regarded as grantees. Thus, protective effects provided by some of these MGEs may have generated an arms race between competing GVs in order to encode the most diverse arsenal of anti-viral weapons, explaining the unusual abundance of MGEs in GV genomes by a kind of ratchet effect. 


\section{Introduction}

Giant Viruses (GVs) compose a remarkable group of eukaryoviruses: prevalent in many environments from the deep ocean to the human gut, they infect a wide spectrum of hosts ranging from tiny protists to vertebrates. They possess highly variable genomes in term of size and content (from $100 \mathrm{~kb}$ to 2,5Mb), play important ecological roles in controlling hosts proliferation and are even suspected to be human pathogens [1-6]. Thus, this peculiar group of virus has been successful in nature and it is important to understand the reasons of this success.

Compared to other eukaryoviruses, GV genomes display two salient features. The first one is the abundance of genes horizontally acquired from cellular organisms. In average, $10 \%$ of the GV genes have been acquired by this process, mainly from prokaryotic sources and occasionally from their hosts [7-11]. The second special feature is the level of gene duplication. For instance, the largest genome of GVs has more than $50 \%$ of its sequence occupied by genes belonging to duplicated families [16].Thus, there is a positive correlation between the GVs genome size and the number of repeated genes [12-15]. Experimental data indicate also that there is a dynamic process of gene duplication and gene loss in response to host adaption [17,18]. All together, importance of horizontal gene transfers and lineage-specific gene duplication have led to the emergence of the «genomic accordion» hypothesis $[12,17,19,20]$ which proposes that GVs adapt to new niches, hosts or changing environments by series of genome contraction and expansion using a combination of gene accretion, duplication and deletion. Whereas this model need to be supported by additional experimental and comparative genomics evidences, the alternative scenario implying a common ancestry with cells has lost some audience. Indeed, the existence of a «fourth kingdom of life» lack reliable phylogenetic signals supporting the kinship of GVs with cells [21] in addition to the absence of genomic proof of a general tendency of GVs genomes to decrease in size and in diversity with time $[7,19,22]$. At the opposite, the « genome accordion » scenario fit perfectly well with a model of genome size evolution observed in birds and mammals under the cross effect of expansion and loss of mobile genetic elements (MGEs) [23].

MGEs form a disparate group of selfish genes that tend to act as molecular parasites into the genomes in which they reside. They are abundant in cellular genomes [24] but rare in the virus world and even almost absent in eukaryovirus [25]. Strikingly, GVs are the exception to this rule. MGEs are diverse and abundant in GV genomes and GVs have apparently cope with this load for a long period of time $[9,15,19]$. In this paper, I collect multiple evidences that several MGEs found in eukaryoviruses have been recruited to perform viral functions. I show that the MGE/GV association has evolved towards cooperation rather than competition, allowing the MGEs to reside in a stable form in the GV genomes. This association, by providing selective advantages to the GVs also 
promote the MGEs dissemination. Thus, I propose the hypothesis that the unusual abundance of MGEs in GV genomes should be explained by the existence of a kind of molecular symbiotic relationship in which both partners benefit from the presence of each other's.

\section{Distribution and origin of MGEs in eukaryoviruses.}

Phylogenies of hallmark genes and phyletic distributions of the genomic repertoires indicated that at least nine GV lineages exist (Figure 1) [26]. Most of these viruses harbor a large diversity of MGEs (Figure 1, Table 1). The majority of them are transposable elements, including diverse Insertion Sequence (IS) families and Eukaryotic-like DNA transposon families as Mariner, PiggyBac or Polinton/Virophage. The last family is of special interest as it clusters a huge diversity of elements including satellite viruses of the GVs (Virophages), composite transposons (Polintons) and their highly-reduced derivatives (Transpovirons) [27-29]. It shall be noted that the capsid protein of the Virophages is related to that of the GVs, suggesting strongly that these elements share a common ancestor [27].

In addition to transposable elements, there are also two kind of Restriction/Modification systems (R-M systems) composed by a gene tandem encoding a restriction endonuclease and a methyltransferase. The endonuclease recognizes and cleaves at specific sites foreign DNA whereas the methyltransferase protects self DNA by transferring methyl groups at the cleavage sites. These systems considered as primitive immune systems in prokaryotes are known to behave as selfish mobile elements. In the case of a system loss, the endonuclease tends to persist in the cytoplasm for a longer period of time compared to the methyltransferase, leading to the restriction of GV and host genomes [30]. R-M systems are not only stably maintained in genomes as addictive selfish modules but also tend to propagate efficiently by lateral gene transfers [31]. Recently, a new class of selfish mobile element has been evidenced in the Bodo saltans virus (Aquavirinae) [26]. The toxin-antitoxin systems (T-A) behave in a similar way as R-M systems and also tend to become addictive modules. In addition, most of GV genomes encode for numerous mobile homing endonucleases frequently associated with self-splicing introns and inteins (also known as protein introns) [9,32,33]. Homing endonucleases are mobile and selfish genes that are known to be invasive, sometimes promoting the «homing» of their cognate introns or inteins into empty sites by the mean of homologous recombination [34].

Finally, more enigmatic MGEs have been evidenced in Mimiviridae: the Major Interspersed Genomic Elements (MIGEs) [32,35]. These elements are composed of a single ORF encoding a protein with a zinc-finger motif preceded by a short piece of non-coding DNA. MIGEs are present in multiple copies (up to 20) and the ORFs have some homologs in a limited number of bacterial 
genomes. Their mode of dissemination is still unknown but they have successfully invaded at least 6 different GV genomes belonging to the Aquavirinae and Mesomimivirinae.

The patchy distribution of most of these MGEs (Figure 1) suggest complex evolutionary histories with multiples events of loss and gain. Most of these MGEs have only prokaryotic homologs suggesting that they have been acquired by lateral transfers from Bacteria or Archaea (Table 1). However, phylogeny of the IS families indicates that GV sequences form monophyletic clusters, suggesting a single event of acquisition for each family [8]. Finally, the genomic comparison of closely related GVs shows the existence of frequent movements and dissemination of these MGEs in addition to vertical and stable transmission for some others ones [19].

Such abundance, diversity and apparent long-standing association of MGEs in GV genomes is a very unusual feature in eukaryoviruses [25]. A possible explanation of this pattern is the existence of reciprocal benefits underlying the association of MGEs with GVs, as suggested by the literature which reveals two kinds of mutualistic association between MGEs and GVs that will be described in the next sections.

\section{Association of MGEs and viruses to degrade host or competing virus genomic DNA.}

The first direct evidence of cooperation of MGEs and GVs has came from the unusual presence of numerous Restriction-Modification systems in Chlorella Phycodnaviruses (Figure 1). These prokaryotic MGEs are known to cooperate with diverse bacterial species in order to degrade invading phage DNA [30]. In Phycodnaviruses, it was shown that the endonuclease of the R-M system is used to degrade immediately after infection the host genomic DNA whereas the methyl-transferase protects the viral DNA [36]. By procuring an abundant source of deoxynucleotides that can be reincorporated into the viral DNA, R-M systems and GVs compose a dual association with an increased global fitness. Do the R-M systems also protect against competing viruses by degradation of their unprotected genomes? So far, this is not evidenced, however in the case of simultaneous infections by two different GVs, a mutual exclusion has been reported in Chlorella Phycodnaviruses [37]. Moreover, the Organic Lake Phycodnavirus (OLPV) encodes a R-M system for which the cognate methyl-transferase, but not the endonuclease, is also encoded by the virophages OLV that are known to infect the OLPVs [38]. Taken together, these data support the view that R-M systems might also be used as anti-viral/anti-virophage agents, leading to an arms race between different co-infecting GVs and/or between GVs and virophages in order to encode the most diverse set of R-M systems. 
GVs also encode numerous introns and inteins that are often associated with a site-specific homing endonuclease. It was demonstrated in diverse bacteriophage groups that these elements are implicated in exclusion processes during competitive infection by two or more viruses [39-41]. By disrupting the enzyme recognition site, the intron prevents self-cleavage by the endonuclease. However, during a mixed infection by two related viruses that differs by the presence or the absence of an endonuclease associated with introns inserted in the same gene, the intron-free genes are specifically cleaved, reducing the fitness of the corresponding virus and favoring the viral genome in which the intron is present [26]. Supporting this view, it's noteworthy that inteins and introns are most often inserted in conserved GV «core» genes that are known to be essential for the achievement of the virus cycle such as DNA polymerase, Ribonucleotide reductase, RNA polymerase or Capsid genes [33,42-44]. Additionally, it was evidenced that the well-known strong site-specificity of the homing-endonuclease is relaxed in GV genomes allowing the inteins to be inserted at multiple sites, in different genes, widening their possible anti-viral effects [32]. These data support the view that the multiplication of introns or inteins in GV genomes are not simply the result of proliferation of selfish genetics elements. They are more likely the consequence of an arm race between competing viruses that have co-opted these genomic parasites as anti-viral weapons.

In this framework, the endonuclease encoded by R-M systems or associated with introns or inteins should be viewed as the anti-viral, anti-virophage or anti-host poison whereas the methyltransferase or the intron or the intein themselves were the antidotes. Incidentally, these MGEs have become addictive modules, applying strong selective pressures favoring their fixation in the viral population and boosting their horizontal dissemination. For these reasons, the association of R-M systems, introns and inteins with the GVs would compose a kind of molecular symbiosis by providing benefits to each partners.

\section{MGE and virus cooperate in order to promote provirus integration}

Viral genome integration into the host genome is a frequent phenomenon in viruses. Indeed, for some viruses, the viral DNA is integrated into the host genome as a transient step of their lifecycle (for example retroviruses) whereas other ones have evolved stable «proviral» versions into host genomes that are transmitted vertically from the parents to the progeny (see for review REF [45]). This process is called endogenisation when the virus has ultimately loss is capability to generate infectious viruses. Interestingly, MGEs seem to have promoted both processes during eukaryovirus evolution. 
Some GVs belonging to the Phycodnarividae family integrated their genomes into their algal host genomes as a biological step of their life cycle [46,47]. The genome sequence of two GVs that generated provirus has revealed the presence of a XerC-like integrase and DDE transposases in both genomes. These enzymes are suspected to catalyze the integration and the excision of the viral DNA into the host genomes [48,49]. Moreover, multiple pro-viral copies of different sizes and inserted at specific GC-CG insertion sites co-exist in the host genomes, suggesting that viral genome integrations are not the result of accidents [47,50]. In this case, the domestication of the integrases/transposases has allowed the viruses to generate a «sleeping» version that is adapted to the peculiar lifestyle of their algal hosts, as Ectocarpale alga have complex life cycles with vegetative and reproductive stages. Viral particles are only released in the environment during the reproductive steps when spores or gametes are generated [51]. If the latency process observed in Ectocarpale viruses is unusual in GVs, the extant of the phenomena might be underestimated as a nearly complete mimiviridae-like virus genome has been found integrated into the Hydra genome [52] and multiple GV genomes fragments have been reported in various cellular genomes [52,53].

The endogenization process should be viewed as the ultimate stage of the latency. The virus has lost its infectious property and behave as selfish DNA as observed for transposons. However, in order to efficiently resides and propagates into the host genome, the virus needs enzymes to catalyze the excision/integration step. Some Herpesviruses, which do not belong to the GV group despite their large genome size (up to $300 \mathrm{~kb}$ ) have resolved the issue by co-opting a transposase derived from a PiggyBac transposable elements [54,55]. The resulting elements called 'Teratorn' lead to large endogenous viral genomes (up to $200 \mathrm{~kb}$ ). They are sometimes surrounded by typical inverted repeats found in PiggyBac transposon that are probably recognized by the transposase to mediate the dissemination of the elements. Teratorn elements have successfully invaded a wide variety of fish genomes in which they are present in high copy number ( $>20$ copies) [56]. Thus, Teratorns provide an additional case of cooperation between a virus and a transposon, in order to successfully invade host genomes by generating endogenous versions of the virus.

A very similar scheme is also observable in Virophages. As previously stated, Virophages are distantly related GV cousins that have in turn became parasites of their relatives. The relationships of Virophages with Polinton and Transpovirons (their miniaturized derivatives) are very complex and look like a network of gene gains and losses [57]. Virophages have the capabilities to insert their genomes both into the GV [29] and the cellular host genome [3,58]. Provirophage genes integrated into the cellular genomes are specifically activated during GV infection conferring an anti-viral protection to the host cells by inhibiting the GV replication. Conversely, it's not known if 
the provirophage integration into the GV genomes increases the fitness of the association by conferring a similar protection against competing virophages for instance. The cooperation between cells and virophages is promoted by the integration and the subsequent dissemination of provirophages into the cell genomes. This process has been achieved by co-opting diverse integrase and transposase genes derived from MGEs. Sputnik-like virophages encode a phage-type, tyrosine recombinase-like integrase and a IS3-like transposase, whereas most Polinton transposons and Mavirus virophages encode a DDE integrase [27,58-60]. Transpoviron genomes do not encode any integrase but an IS3-like transposase gene is present. This transposase has some similarities with the Sputnik IS3 transposases [29]. The mutualistic association between virophage-like elements and MGE genes domesticated to mediate provirophage integration/dissemination provide an additional remarkable example of tripartite cooperation between the host cell, the virophage and the domesticated integrase/transposase genes in order to suppress viral infection, increasing the fitness of the symbiotic system.

\section{Conclusion}

The abundance and the diversity of MGEs is one of the most salient features of the GVs genomes. The successful invasion of these MGEs should be explained by the existence of a kind of molecular symbiosis: while the GV provides a niche in which the MGEs are stably maintained and vertically transmitted in the viral progeny, MGE genes has been used to fulfill viral functions as defense against competing viruses, degradation of the host DNA or the generation of provirus able to insert and reside into the host genomes. By providing competitive advantages to the GV, the MGEs inserted into the GV genomes also increase their own fitness. However, R-M systems and intron/intein associated with homing endonucleases may also became addictive as the loss of the methyltransferase genes or the introns/inteins would no longer protect the viral genome against the deleterious effect of the corresponding site-specific restriction enzymes. Competition between different viruses infecting the same host may also lead to an arm race in which each virus have deployed an arsenal of anti-viral compounds. As new MGE families are frequently discovered in GVs genomes [35,61] new kinds of association may be discovered in the next future providing further supports to the existence of molecular symbiosis between these genetic entities.

\section{Conflict of interest}

The author declares no conflict of interest. 


\section{Acknowledgments}

Jean-Michel Rossignol for providing useful comments to the manuscript.

\section{References}

1. Boughalmi M, Pagnier I, Aherfi S, Colson P, Raoult D, La Scola B: First isolation of a giant virus from wild Hirudo medicinalis leech: Mimiviridae isolation in Hirudo medicinalis. Viruses 2013, 5:2920-2930.

2. Campos RK, Boratto PV, Assis FL, Aguiar ER, Silva LC, Albarnaz JD, Dornas FP, Trindade GS, Ferreira PP, Marques JT: Samba virus: a novel mimivirus from a giant rain forest, the Brazilian Amazon. Virology journal 2014, 11:95.

3. Maumus F, Epert A, Nogué F, Blanc G: Plant genomes enclose footprints of past infections by giant virus relatives. Nature communications 2014, 5:ncomms5268.

4. Monier A, Claverie J-M, Ogata H: Taxonomic distribution of large DNA viruses in the sea. Genome biology 2008, 9:R106.

5. Yolken RH, Jones-Brando L, Dunigan DD, Kannan G, Dickerson F, Severance E, Sabunciyan S, Talbot CC, Prandovszky E, Gurnon JR: Chlorovirus ATCV-1 is part of the human oropharyngeal virome and is associated with changes in cognitive functions in humans and mice. Proceedings of the National Academy of Sciences 2014, 111:16106-16111.

6. Claverie J-M, Grzela R, Lartigue A, Bernadac A, Nitsche S, Vacelet J, Ogata H, Abergel C: Mimivirus and Mimiviridae: giant viruses with an increasing number of potential hosts, including corals and sponges. Journal of invertebrate pathology 2009, 101:172-180.

7. Moreira D, Brochier-Armanet C: Giant viruses, giant chimeras: the multiple evolutionary histories of Mimivirus genes. BMC Evolutionary Biology 2008, 8:12.

8. Filée J: Lateral gene transfer, lineage-specific gene expansion and the evolution of Nucleo Cytoplasmic Large DNA viruses. Journal of invertebrate pathology 2009, 101:169-171.

9. Filée J, Siguier P, Chandler M: I am what I eat and I eat what I am: acquisition of bacterial genes by giant viruses. Trends in Genetics 2007, 23:10-15.

10. Iyer LM, Balaji S, Koonin EV, Aravind L: Evolutionary genomics of nucleo-cytoplasmic large DNA viruses. Virus research 2006, 117:156-184.

11. Yutin N, Koonin EV: Hidden evolutionary complexity of Nucleo-Cytoplasmic Large DNA viruses of eukaryotes. Virology journal 2012, 9:161.

12. Filée J: Route of NCLDV evolution: the genomic accordion. Current opinion in virology 2013, 3:595-599.

13. Filée J, Chandler M: Convergent mechanisms of genome evolution of large and giant DNA viruses. Research in microbiology 2008, 159:325-331. 
14. Suhre K: Gene and genome duplication in Acanthamoeba polyphaga Mimivirus. Journal of virology 2005, 79:14095-14101.

15. Shukla A, Chatterjee A, Kondabagil K: The number of genes encoding repeat domain-containing proteins positively correlates with genome size in amoebal giant viruses. Virus evolution 2018, 4:vex039.

* This paper offers an updated panorama of the diversity and the evolution of repeat sequences in Giant Viruses.

16. Legendre M, Fabre E, Poirot O, Jeudy S, Lartigue A, Alempic J-M, Beucher L, Philippe N, Bertaux L, Labadie K: Diversity and evolution of the emerging Pandoraviridae family. bioRxiv 2017,

17. Elde NC, Child SJ, Eickbush MT, Kitzman JO, Rogers KS, Shendure J, Geballe AP, Malik HS: Poxviruses deploy genomic accordions to adapt rapidly against host antiviral defenses. Cell 2012, 150:831-841.

** This article demonstrates that Poxviruses evolved trough a balanced process of gene duplication and gene loss to adapt to the host defense systems.

18. Boyer M, Azza S, Barrassi L, Klose T, Campocasso A, Pagnier I, Fournous G, Borg A, Robert $\mathrm{C}$, Zhang X: Mimivirus shows dramatic genome reduction after intraamoebal culture. Proceedings of the National Academy of Sciences 2011, 108:10296-10301.

19. Filée J: Genomic comparison of closely related Giant Viruses supports an accordion-like model of evolution. Frontiers in microbiology 2015, 6:593.

20. Schulz F, Yutin N, Ivanova NN, Ortega DR, Lee TK, Vierheilig J, Daims H, Horn M, Wagner $\mathrm{M}$, Jensen GJ: Giant viruses with an expanded complement of translation system components. Science 2017, 356:82-85.

21. Williams TA, Embley TM, Heinz E: Informational gene phylogenies do not support a fourth domain of life for nucleocytoplasmic large DNA viruses. PLoS One 2011, 6:e21080.

22. Yutin N, Wolf YI, Koonin EV: Origin of giant viruses from smaller DNA viruses not from a fourth domain of cellular life. Virology 2014, 466:38-52.

23. Kapusta A, Suh A, Feschotte C: Dynamics of genome size evolution in birds and mammals. Proceedings of the National Academy of Sciences 2017, 114:E1460-E1469.

24. Hua-Van A, Le Rouzic A, Boutin TS, Filée J, Capy P: The struggle for life of the genome's selfish architects. Biology direct 2011, 6:19.

25. Gilbert C, Cordaux R: Viruses as vectors of horizontal transfer of genetic material in eukaryotes. Current opinion in virology 2017, 25:16-22.

26. Deeg CM, Chow C-ET, Suttle CA: The kinetoplastid-infecting Bodo saltans virus (BsV), a window into the most abundant giant viruses in the sea. bioRxiv 2017,

27. Koonin EV, Krupovic M: Polintons, virophages and transpovirons: a tangled web linking viruses, transposons and immunity. Current opinion in virology 2017, 25:7-15. 
** This papers offers a state-of-the-art of the evolutionary connections between virophages and various transposable elements found in eukaryotes and in Giant Viruses.

28. Krupovic M, Koonin EV: Polintons: a hotbed of eukaryotic virus, transposon and plasmid evolution. Nature Reviews Microbiology 2015, 13:105.

29. Desnues C, La Scola B, Yutin N, Fournous G, Robert C, Azza S, Jardot P, Monteil S, Campocasso A, Koonin EV: Provirophages and transpovirons as the diverse mobilome of giant viruses. Proceedings of the National Academy of Sciences 2012, 109:18078-18083.

* This paper reports the discovery of the transpovirons, a specific class of mobile genetic elements present in Giant Virus genomes.

30. Kobayashi I: Behavior of restriction-modification systems as selfish mobile elements and their impact on genome evolution. Nucleic acids research 2001, 29:3742-3756.

31. Vasu K, Nagaraja V: Diverse functions of restriction-modification systems in addition to cellular defense. Microbiology and molecular biology reviews 2013, 77:53-72.

32. Gallot-Lavallée L, Blanc G, Claverie J-M: Comparative genomics of chrysochromulina ericina virus and other microalga-infecting large DNA viruses highlights their intricate evolutionary relationship with the established Mimiviridae family. Journal of virology 2017, 91:e00230-17.

33. Ogata H, Raoult D, Claverie J-M: A new example of viral intein in Mimivirus. Virology journal 2005, 2:8.

34. Stoddard BL: Homing endonuclease structure and function. Quarterly reviews of biophysics 2005, 38:49-95.

35. Santini S, Jeudy S, Bartoli J, Poirot O, Lescot M, Abergel C, Barbe V, Wommack KE, Noordeloos AA, Brussaard CP: Genome of Phaeocystis globosa virus PgV-16T highlights the common ancestry of the largest known DNA viruses infecting eukaryotes. Proceedings of the National Academy of Sciences 2013, 110:10800-10805.

36. Agarkova IV, Dunigan DD, Van Etten JL: Virion-associated restriction endonucleases of chloroviruses. Journal of virology 2006, 80:8114-8123.

37. Chase TE, Nelson JA, Burbank DE, van Etten JL: Mutual exclusion occurs in a chlorella-like green alga inoculated with two viruses. Journal of general virology 1989, 70:1829-1836.

38. Yau S, Lauro FM, DeMaere MZ, Brown MV, Thomas T, Raftery MJ, Andrews-Pfannkoch C, Lewis M, Hoffman JM, Gibson JA: Virophage control of antarctic algal host-virus dynamics. Proceedings of the National Academy of Sciences 2011, 108:6163-6168.

39. Zeng Q, Bonocora RP, Shub DA: A free-standing homing endonuclease targets an intron insertion site in the psbA gene of cyanophages. Current biology 2009, 19:218-222.

40. Belle A, Landthaler M, Shub DA: Intronless homing: site-specific endonuclease SegF of bacteriophage T4 mediates localized marker exclusion analogous to homing endonucleases of group I introns. Genes \& development 2002, 16:351-362. 
41. Goodrich-Blair H, Shub DA: Beyond homing: competition between intron endonucleases confers a selective advantage on flanking genetic markers. Cell 1996, 84:211-221.

42. Nagasaki K, Shirai Y, Tomaru Y, Nishida K, Pietrokovski S: Algal viruses with distinct intraspecies host specificities include identical intein elements. Applied and environmental microbiology 2005, 71:3599-3607.

43. Bigot Y, Piégu B, Casteret S, Gavory F, Bideshi DK, Federici BA: Characteristics of inteins in invertebrate iridoviruses and factors controlling insertion in their viral hosts. Molecular phylogenetics and evolution 2013, 67:246-254.

44. Fischer MG, Allen MJ, Wilson WH, Suttle CA: Giant virus with a remarkable complement of genes infects marine zooplankton. Proceedings of the National Academy of Sciences 2010, 107:19508-19513.

45. Aswad A, Katzourakis A: Paleovirology: The Study of Endogenous Viral Elements. Virus Evolution: Current Research and Future Directions; Weaver, SC, Denison, $M$, Roossinck, $M$, Vignuzzi, M, Eds 2016,

46. Delaroque N, Maier I, Knippers R: Persistent virus integration into the genome of its algal host, Ectocarpus siliculosus (Phaeophyceae). Journal of General Virology 1999, 80:1367-1370.

47. Delaroque N, Boland W: The genome of the brown alga Ectocarpus siliculosus contains a series of viral DNA pieces, suggesting an ancient association with large dsDNA viruses. BMC evolutionary biology 2008, 8:110.

48. Delaroque N, Müller DG, Bothe G, Pohl T, Knippers R, Boland W: The complete DNA sequence of the Ectocarpus siliculosus virus EsV-1 genome. Virology 2001, 287:112-132.

49. Schroeder DC, Park Y, Yoon H-M, Lee YS, Kang SW, Meints RH, Ivey RG, Choi T-J: Genomic analysis of the smallest giant virus-Feldmannia sp. virus 158. Virology 2009, 384:223-232.

50. Meints RH, Ivey RG, Lee AM, Choi T-J: Identification of two virus integration sites in the brown alga Feldmannia chromosome. Journal of virology 2008, 82:1407-1413.

51. Van Etten JL, Meints RH: Giant viruses infecting algae. Annual Reviews in Microbiology 1999, 53:447-494.

52. Filée J: Multiple occurrences of giant virus core genes acquired by eukaryotic genomes: The visible part of the iceberg? Virology 2014, 466:53-59.

53. Gallot-Lavallée L, Blanc G: A glimpse of nucleo-cytoplasmic large DNA virus biodiversity through the eukaryotic genomics window. Viruses 2017, 9:17.

54. Aswad A, Katzourakis A: A novel viral lineage distantly related to herpesviruses discovered within fish genome sequence data. Virus evolution 2017, 3:vex016.

55. Inoue Y, Saga T, Aikawa T, Kumagai M, Shimada A, Kawaguchi Y, Naruse K, Morishita S, Koga A, Takeda H: Complete fusion of a transposon and herpesvirus created the Teratorn mobile element in medaka fish. Nature communications 2017, 8:551. 
** This article describes the cooperation of PibbyBac transposons and Herpesviruses to promote the endogenization of the viruses.

56. Inoue Y, Kumagai M, Zhang X, Saga T, Wang D, Koga A, Takeda H: Fusion of piggyBac-like transposons and herpesviruses occurs frequently in teleosts. Zoological letters 2018, 4:6.

57. Yutin N, Raoult D, Koonin EV: Virophages, polintons, and transpovirons: a complex evolutionary network of diverse selfish genetic elements with different reproduction strategies. Virology journal 2013, 10:158.

58. Fischer MG, Hackl T: Host genome integration and giant virus-induced reactivation of the virophage mavirus. Nature 2016, 540:288.

** This article demonstrates that virophages and cells have co-evolved to develop an anti-viral defense mechanism against Giant Viruses.

59. La Scola B, Desnues C, Pagnier I, Robert C, Barrassi L, Fournous G, Merchat M, Suzan-Monti M, Forterre $\mathrm{P}$, Koonin $\mathrm{E}$ : The virophage as a unique parasite of the giant mimivirus. Nature 2008, 455:100.

60. Roux S, Chan L-K, Egan R, Malmstrom RR, McMahon KD, Sullivan MB: Ecogenomics of virophages and their giant virus hosts assessed through time series metagenomics. Nature communications 2017, 8:858.

61. Sun C, Feschotte C, Wu Z, Mueller RL: DNA transposons have colonized the genome of the giant virus Pandoravirus salinus. BMC biology 2015, 13:38.

*This article describes an atypical family of transposable elements found in Giant Viruses with evidence of domestication events.

Figure Legends

Figure 1: Diversity of mobile genetic elements found in Giant Virus genomes. The heat-map represents the maximum number of copies of each family of mobile elements identified in each viral lineages. The phylogeny is a consensual tree obtained with conserved viral core genes. 


\begin{tabular}{|c|c|c|c|c|}
\hline \multicolumn{2}{|l|}{ Mobile Genetic Elements } & Distribution & Origin & Biological roles \\
\hline & IS4-like & Phycodnaviridae EsV-1 and FirrV-1 & Prokaryote & \\
\hline Insertion Sequence & IS607 & Phycodnaviridae, Mimiviridae, Asfanviridae & Prokaryote & Genome integration into the host/viral genome \\
\hline & $\begin{array}{c}\text { IS5 } \\
\text { IS3-like }\end{array}$ & $\begin{array}{c}\text { Aquavirinae } \\
\text { Vironhe Mavirus Transnoviron }\end{array}$ & Prokaryote & \\
\hline \multicolumn{2}{|l|}{ Restriction-Modification system } & Phycodnaviridae, Mimiviridae, Marseilleviridae / virophage OLV & Prokaryote & Host DNA degradation, Competing virus / virophage DNA degradation \\
\hline \multirow{3}{*}{ Intein and Intron-associated endonuclease } & $\mathrm{HNH}$ & Mimiviridae, Phycodnaviridae, Asfarviridae, Marseilleviridae & Prokaryote & \\
\hline & GIY-YIG & Iridoviridae, Phycodnaviridae, Mesomimivirinae, Asfarviridae & Prokaryote & Cleave competing virus DNA \\
\hline & LAGLIDADG & Mesomimiviridae, Iridoviridae & Prokaryote & \\
\hline \multicolumn{2}{|l|}{ Toxin/Antitoxin } & Bodo saltans virus & Prokaryote & \\
\hline \multirow{3}{*}{ DNA transposon } & Mariner & Pandoraviridae, Iridoviridae & Eukaryote & Unkown but several copies have been domesticated \\
\hline & Mutator & (Baculovirus) & Eukaryote & \\
\hline & PiggyBac & Iridoviridae, (Herpesviridae) & Eukaryote & Endogenization \\
\hline \multicolumn{2}{|c|}{ Virophage/Polinton/Transpoviron } & Mimiviridae, Phycodnaviridae & Eukaryote & Host anti-viral protection \\
\hline \multicolumn{2}{|c|}{ Major Interspersed Genomic Element (MIGE) } & Mesomimivirinae, Aquavirinae & Prokaryote (?) & \\
\hline
\end{tabular}

\section{Table 1: Distribution, origin and possible biological roles of mobile genetic elements found in virus genomes}



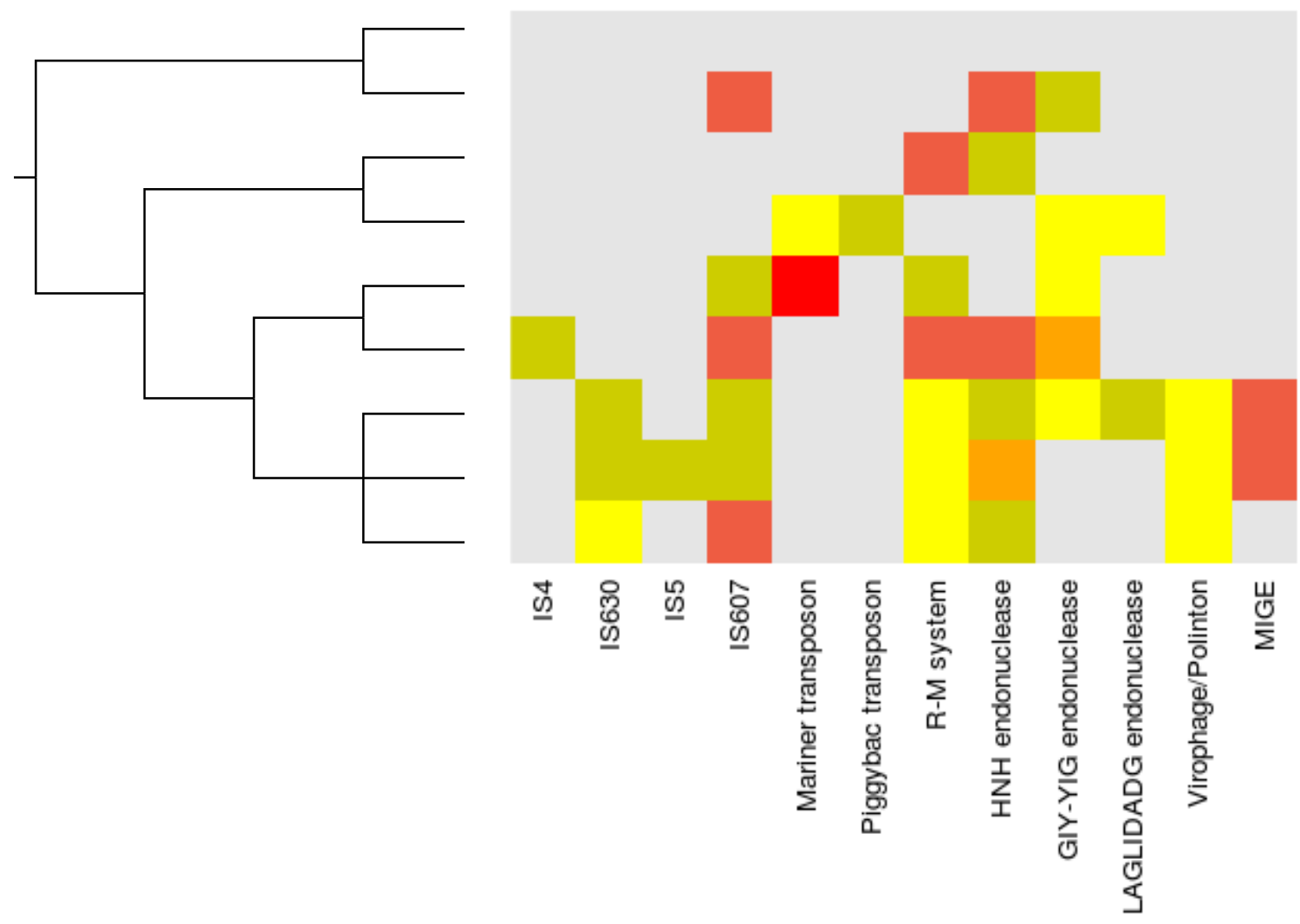

Poxviridae

Asfarviridae

Marseilleviridae

Iridoviridae

Pandoraviridae

Phycodnaviridae

Mesomimiviridae

Aquavirinae

Megavirinae

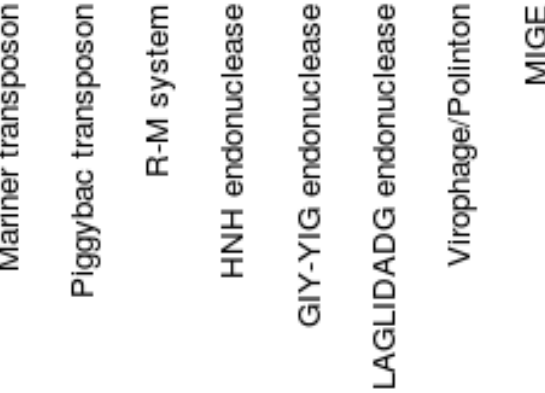

